\title{
Heat Stress-Induced EGF Receptor Transactivation in A431 Cells
}

\author{
A. L. Evdonin, D. A. Popova, and N. D. Medvedeva \\ Institute of Cytology, Russian Academy of Sciences, Tikhoretskii prosp., 4, St. Petersburg, 164064 Russia; \\ e-mail:evdonin@mail.ru
}

DOI: $10.1134 / \mathrm{S} 1990747809030180$

Ligand-independent EGF receptor (EGFR) activation is referred to as EGFR transactivation. EGFR transactivation was shown to be induced by agonists of G-protein coupled receptors or cytokine receptors as well as stressful factors. The mechanism of EGFR transactivation following G-protein coupled receptors activation is actively investigated. The molecular factor, which acts as signal transduction activator under stress conditions (heat shock, oxidative stress, ionizing radiation, etc.) is currently unknown.

Previously we have shown that heat shock protein 70 (Hsp70) is released from A431 cells at initial steps of heat shock. We hypothesized that this protein induces EGFR transactivation.

We have found that EGFR undergoes tyrosine phosphorylation during incubation with conditioned medium of heated cells, whereas the depletion of Hsp70 from conditioned medium of heated cells abrogates EGFR transactivation, which suggests that Hsp70 participates in EGFR transactivation at initial steps of heat stress.

This notion is supported by the findings that purified Hsp70 can induce EGFR transactivation and the activation of EGFR-dependent signaling pathways. Both heat stress and pure Hsp70 stimulate activation of TLR2/4 and their association with EGFR. Neutralizing antibodies against TLR2/4 decrease Hsp70-induced EGFR activation.

These results suggest that the secreted Hsp70 stimulates EGFR transactivation in heat stressed A431cells by cross-communication of TLR and EGFR signaling systems. 